\title{
Réalisation variable des occlusives en français louisianais : l'affrication et l'aspiration à la Ville Platte
}

\author{
Alexander Russell \\ Université Western Ontario \\ arusse25@uwo.ca
}

\section{Introduction}

Les origines des groupes colonisateurs étant très diverses, le français parlé en Louisiane fait preuve de beaucoup de variation dialectale dans la réalisation des phonèmes. L'influence acadienne est bien établie, mais les Acadiens ne constituent qu'une partie de la francophonie louisianaise. Des gens venus directement de France et d'autres venus du Canada ont laissé des traces dans le français entendu aujourd'hui. Ce qui suit est une analyse des consonnes occlusives observées dans des extraits d'entrevues faites avec des locuteurs natifs de la région de la Ville Platte en Louisiane. Nous considérons dans cet article les occurrences de deux phénomènes, l'affrication et l'aspiration, et comment ces phénomènes s'influencent (si l'aspiration empêche l'affrication par exemple) et sont influencés par l'accentuation des syllabes, leur position dans le mot et la langue anglaise. Nous montrons que l'affrication, un phénomène qui est très rare en Louisiane, est presque systématique dans le français de la région qui entoure la Ville Platte dans les prairies louisianaises, et que l'aspiration caractéristique de l'anglais a profondément pénétré cette variété de français.

\section{Les occlusives dans le français de la Ville Platte}

L'étude des occlusives est particulièrement intéressant puisque, selon Picone et Valdman (2005:146), le dialecte de la région de Ville Platte est reconnu pour l'affrication des dentales ([ts] et [dz]), qui est un trait caractéristique des parlers laurentiens et peu connu ailleurs en Louisiane. En ce qui concerne l'anglais, on sait qu'il a pénétré le lexique du français louisianais (Klingler et al. 1997:160), mais influence-t-il la prononciation des mots français aussi ? L'aspiration des occlusives sourdes typique de l'anglais est un trait particulièrement intéressant, puisque Dubois et Horvath (1998) ont déjà souligné son absence dans l'anglais de la Louisiane. La présence de l'affrication et de l'aspiration à la Ville Platte sera le point principal de cette étude.

\subsection{Les occlusives affriquées}

L'affrication des occlusives dentales /t/ et /d/ devant les voyelles antérieures et fermées /i/ et /y/ et les semi-consonnes $/ \mathrm{j} /$ et $/ \mathrm{u} /$ est un trait caractéristique du français québécois et des dialectes du français nord-américain qui sont issus de la vallée laurentienne, mais elle est peu répandue en Louisiane. Ce phénomène est observé surtout dans la paroisse d'Évangeline et est typique de la variété parlée à la Ville Platte (Picone et Valdman 2005:146; Poirier 2009:390). Le spectrogramme suivant nous montre l'affrication d'une occlusive dentale. 


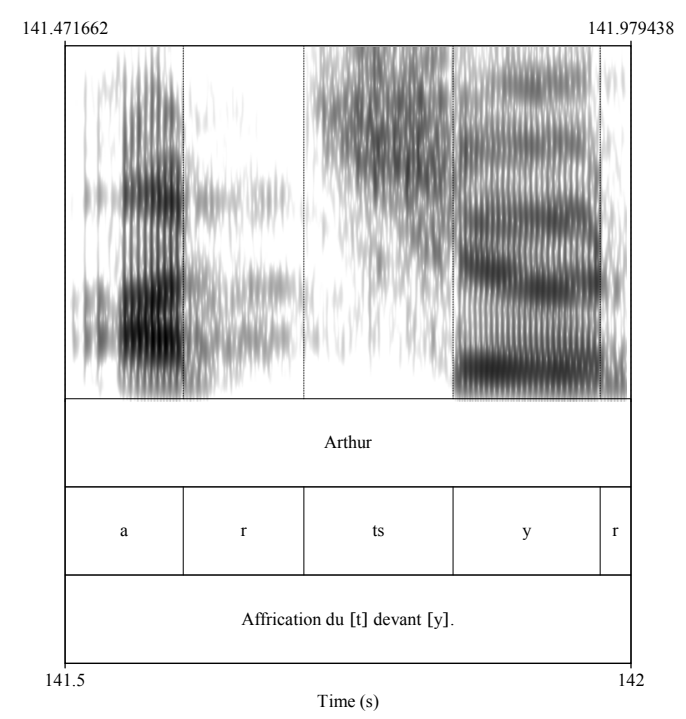

Figure 1 : Spectrogramme de l'affrication. L'affrication de l'occlusive dentale sourde $[t]$ devant la voyelle antérieure fermée [y] dans le mot « Arthur ». (loc. F1, 142s)

Poirier décrit le phénomène comme «un relâchement progressif de l'occlusion, ce qui a pour effet d'allonger la durée de la portion finale de la consonne (la détente), d'où la perception d'un fort bruit fricatif» (2009:376). Selon Laver, lorsqu'une occlusive est suivie d'une sonante, le relâchement d'air pendant le chevauchement des deux segments peut être prolongé. Cette prolongation permet à l'articulateur actif de passer plus lentement à travers la zone d'approximation fermée avant d'atteindre la position de la sonante, ce qui a pour résultat une friction audible de durée perceptible au même lieu d'articulation que l'occlusive (1994:363).

Deux types d'affriquées ont été observés dans les français nord-américains, dont la présence dépende du dialecte. Le premier cas est celui où « la portion constrictive correspond à une chuintante (comme dans les prononciations acadiennes [t $\mathrm{f}]$ et $[\mathrm{d} 3]$ ) » et l'autre est celui où elle correspond à une sifflante (comme dans les prononciations québécoises [ts] et [dz]) (Poirier 2009:377). Nous avons déjà mentionné la présence des formes québécoises à la Ville Platte, et en ce qui concerne les formes acadiennes, Papen et Rottet (1997) indiquent dans leur étude du dialecte du bassin Lafourche que, pour un groupe de mots limité, les occlusives vélaires ont des variantes affriquées alvéo-palatales devant les voyelles antérieures et la semi-consonne [j] comme dans $/ \mathrm{dj} ø />[\mathrm{d} 3 ø]$ « dieu » et dans $/ \operatorname{kadj} \tilde{\varepsilon} />[\operatorname{kad} 3 \tilde{\varepsilon}]$ « Cadien ». Ces deux formes sont présentes dans notre corpus de locuteurs originaires de la Ville Platte.

(1) Char bon dieu. (loc. F2, 547s)

(2) Ici avec les Cadiens... (loc. M3, 530s)

Un autre type d'affrication observée dans notre corpus est de type anglais. Laver (1994:366) indique que dans bien des accents anglais, les groupes / $\mathrm{tr} /$ et / $\mathrm{dr} /$ en position initiale de syllabe sont prononcés comme des affriquées post-alvéolaires [t $\mathrm{t} \lambda]$ et [d3」].

(3) Big country station. (loc. M1, 172s)

(4) Quelques drinks. (loc. F2, 470s)

Poirier (2009:378-379) a noté que dans le cas où la partie constrictive de l'affrication devient une sifflante, l'affrication «se produit impérativement à l'intérieur d'un mot dans l'usage québécois » mais 
devient facultative quand il y a une frontière de mot entre la consonne et la voyelle qui suit. Nous verrons que, dans notre corpus, l'affrication à l'intérieur d'un mot est presque catégorique tandis que l'affrication entre deux mots n'est observée qu'une seule fois.

\subsection{Les occlusives aspirées}

L'aspiration est un phénomène phonétique qui ne fait pas partie, normalement, du répertoire phonologique français (Corneau 2000:44) et le français louisianais ne fait pas exception. Bien que l'aspirée glottale [h] existe en Louisiane (Picone et Valdman 2005:151; Papen et Rottet 1997:76), nous n'avons trouvé aucune source qui mentionne l'aspiration des consonnes occlusives. Dans un article sur les fricatives interdentales en anglais cadien, Dubois et Horvath mentionnent que l'absence de l'aspiration des consonnes $/ \mathrm{p}, \mathrm{t}, \mathrm{k} / \mathrm{est}$ un trait considéré typique de l'anglais cadien. Elles soulignent que la source de ce trait chez des locuteurs cadiens bilingues est normalement considérée comme le résultat de l'interférence du français cadien tout en indiquant que cela n'a pas encore été confirmé (1998:246). Si nous supposons que l'interférence entre les deux langues est probable, et nous considérons le fait que nos quatre locuteurs ont grandi pendant une période où ils ont subi «de fortes pressions économiques et sociales en faveur de l'anglais » (Dubois 2005:289), il serait raisonnable de croire que cette influence peut agir dans l'autre sens aussi.

L'aspiration des occlusives sourdes, /p/, /t/ et / $/$ / est un phénomène typiquement anglais, qui ne sous-tend pas de contraste phonémique évident dans des mots tels que pin [phin], et tonne [th^n]. L'aspiration est une poussée d'air audible qui accompagne l'articulation de certaines occlusives consonantiques lors du relâchement du son (Crystal 2008:38). Le relâchement ressemble à une fricative brève réalisée au même lieu que l'articulation de l'occlusive, et l'aspiration est un son sourd qui partage bien des traits de la voyelle qui le suit (Ladefoged 2003:158). Cette aspiration est la plus forte au début des syllabes accentuées, mais peut être aussi présente, bien que plus faiblement, dans l'attaque des syllabes non accentuées. Après la fricative [s] dans des mots tels que «stay» ou «spade» cependant, il n’y a pas d'aspiration (Crystal 2008:39). La figure 2 nous montre que, dans un spectrogramme, on peut voir la frication associée avec l'aspiration de l'occlusive entre l'explosion de celle-ci et le voisement de la voyelle qui la suit.

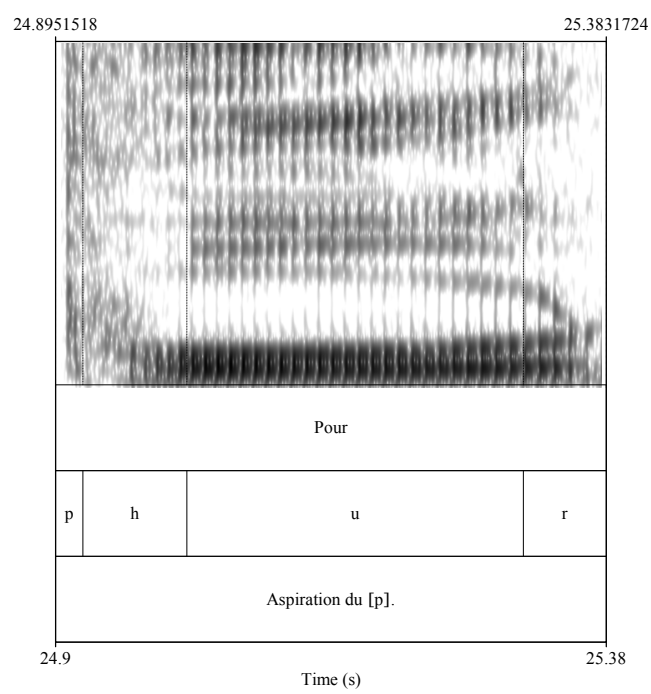

Figure 2 : Spectrogramme de l'aspiration. L'aspiration de l'occlusive sourde [p] dans le mot «pour». (loc. M1, 25s) 
Selon le raisonnement de Laver, qui est de l'avis que le relâchement final d'un énoncé n'est pas un cas d'aspiration, le relâchement final concerne une relation coordonnée entre une occlusive et un silence à la fin d'un énoncé et pas entre deux segments à l'intérieur d'un énoncé (1994:355-356). Dans ce travail nous partageons l'opinion de Laver et pas celle de Davenport et Hannahs qui considèrent ce relâchement comme un cas d'aspiration (1998:23).

\subsection{La présence des mots anglais en français louisianais}

Dans cette section, nous établirons les critères qui nous permettront de distinguer l'emprunt des mots anglais de l'alternance codique dans un corpus de français louisianais. Nous avons déjà mentionné l'influence prépondérante de l'anglais sur le français louisianais. Cette influence date de l'achat de la Louisiane par les États-Unis en 1803 il y a plus de deux siècles (Picone et Valdman 2005:157). Dans une étude sur les pratiques bilingues en Louisiane, Dubois et al. ont souligné que :

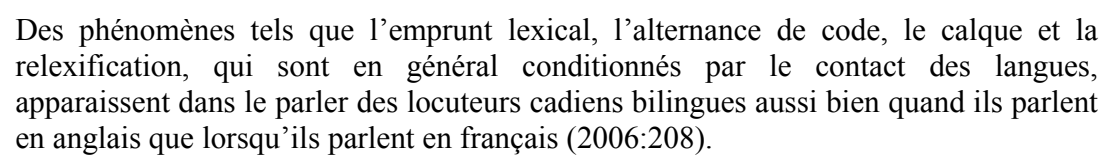

Étant donné que nos locuteurs sont tous bilingues, il suffit de dire qu'ils subissent les mêmes influences de l'anglais que nous venons de mentionner. En étudiant une variété de français dont tous les locuteurs sont bilingues et emploient régulièrement des emprunts, il est important d'établir quels mots sont intégrés au français et quels mots ne le sont pas. Afin d'exécuter cette tâche, nous avons dû considérer la phonologie, l'accentuation et le contexte du mot.

Picone et Valdman indiquent qu'il existe un groupe d'emprunts qui «sont pleinement assimilés et font partie intégrante du lexique de la langue » (2005:158), mais ces emprunts ont tendance à dater d'il y a longtemps. Afin de déterminer si un mot était un emprunt nous avons consulté le dictionnaire de J. O. Daigle (1993), bien que Klingler et al. (1997:147) avouent que l'œuvre manque «de rigueur méthodologique », et nous avons considéré la prononciation et l'intonation. Klingler et al. indiquent qu'à cause du bilinguisme sociétal, tout le monde comprend un item lexical anglais dans sa forme non assimilée (1997:174) et Picone et Valdman soulignent que «l'emprunt est devenu une stratégie lexicogénique peu nécessaire et moins fréquente, car il est toujours possible [...] d'insérer [...] un élément anglais inassimilé (lexème ou syntagme)» (2005:159). Dubois et al. ont découvert que «près de $77 \%$ des mots d'origine anglaise ne sont pas intégrés phonologiquement » en ajoutant que presque tous les mots grammaticaux (particules, prépositions et conjonctions) ne montrent aucune intégration (2006:209). Ces mots sont clairement anglais et sont considérés comme tel. Afin d'être intégrés, les noms pluriels doivent être non fléchis (la pluralité se manifestant par le déterminant) et elles ont trouvé que $72 \%$ des noms pluriels sont intégrés (2006:210). Nous avons constaté cette même intégration dans notre corpus (cf. exemple 5), mais nous avons quand même considéré ces mots comme étant anglais à cause de la prononciation.

(5) Un de ses classemates... ['klasmeIt] (loc. M1, 287s)

Picone et Valdman mentionnent aussi la présence des calques qui « prennent la forme de modélisation sur un homologue anglais, que ce soit un lexème, par ex. récorder « enregistrer » (to record) (cf. exemple 6) [...] ou un syntagme» (2005:158), et ces mots sont considérés comme français à cause de la prononciation française.

(6) Il avait récordé leurs, leurs voix. [recכr'de] (loc. M1, 69s) 
Plusieurs chercheurs soulignent le fait que ceux qui parlent le FC couramment ne fléchissent pas les mots anglais utilisés comme participe passé afin de former le passé composé (Blyth 1997:41; Klingler et al. 1997:174-175) et de plus, Picone et Valdman indiquent que dans ces cas « il n'y a aucun effort d'adapter la prononciation qui demeure celle de l'original anglais » (2005:159). Par conséquent, ces mots seront jugés anglais. Concernant l'accentuation, Lyche précise que le FC «se distingue très nettement du français standard (FS) pour ce qui est de la longueur de la phrase phonologique » celle-ci étant plus courte en FC (1996:42). Cette différence est attribuée au fait que le FC «peut accentuer chaque unité lexicale » et l'accent de mot se rapproche de celui de l'anglais (1996:42). Elle remarque aussi qu'en FC l'accent «peut frapper la dernière syllabe d'un groupe, mais il peut aussi, comme en anglais, frapper l'une des syllabes d'un mot et pas obligatoirement la première comme sous l'accent initial» (1996:44). Lyche propose que le détachement du FC au niveau de l'accentuation et du rythme puisse être le résultat d'une influence de l'anglais, mais elle note en outre, la présence de certains de ces traits suprasegmentaux dans le FS de nos jours (1996:44). Dans le cadre de cette étude, en ce qui concerne les calques comme ceux d'exemple (6) et les emprunts, ainsi que les noms propres, le mot est considéré comme anglais si l'accentuation et la prononciation suivent les règles anglaises. Si un mot est accentué sur la syllabe finale en anglais et la prononciation n'est pas clairement anglaise, le mot est considéré comme français.

\section{Méthodologie}

Le corpus est constitué d'enregistrements de quatre locuteurs de la Ville Platte, une communauté dans une région reconnue pour l'affrication des dentales [t] et [d]. Il s'agit de deux hommes (M1: 69 ans; M3 : 64 ans) et deux femmes (F1: 76 ans; F2: 87 ans), tous natifs de cette communauté. Les enregistrements analysés sont de type spontané, durent environ 10 minutes chacun et sont extraits d'une série d'entretiens plus longs. Ils ont été réalisés entre le 17 et le 19 novembre 2007 par Tom Klingler de l’Université Tulane.

Ces quatre locuteurs sont nés dans la première moitié du siècle dernier et ont grandi dans des familles francophones. Ils n'ont pas tous grandi à la Ville Platte et certains ont passé leur enfance un peu partout dans l'arrière-pays de la ville. La plus grande partie de leur vie s'est déroulée dans les prairies louisianaises, surtout dans la paroisse Évangéline où se situe la Ville Platte. Leur niveau d'éducation varie, mais aucun n'a fréquenté l'école française. Ce n'est qu'avec des membres de leur famille d'une génération similaire et des amis proches qu'ils parlent encore français.

Nous avons analysé la présence de l'affrication et de l'aspiration et nous avons comparé la fréquence de chacun de ces phénomènes chez chaque locuteur à l'aide du logiciel Praat (http://www.fon.hum.uva.nl/praat/: 17/12/09). Nous avons relevé pour chaque occlusive, la position dans le mot (début, fin, ailleurs) ainsi que la présence d'un accent sur la syllabe dans laquelle elle est réalisée. Nous avons aussi noté si cette occlusive est suivie d'une voyelle ou d'une consonne (et le type de voyelle en ce qui concerne l'affrication), et la présence ou l'absence (si les conditions phonétiques permettant la présence d'un certain phénomène sont atteintes) d'une variété de phénomènes phonétiques.

Nous avons employé le spectrogramme du logiciel Praat afin de déterminer si une consonne est affriquée ou aspirée. Étant donné que seules les sourdes peuvent être aspirées, toute activité identifiable à un «bruit» sur le spectrogramme entre un /d/ et une voyelle a été considérée comme la réalisation de l'affrication. De même, nous avons mentionné que ce phénomène n'est possible qu'après les dentales, ce qui nous permet de considérer l'activité réalisée entre les occlusives $/ \mathrm{p} /$ et $/ \mathrm{k} /$ et les voyelles qui les suivent comme de l'aspiration. Reste le cas de /t/ qui peut sembler problématique étant donné son lieu d'articulation et l'absence de voisement qui le caractérise. Dans le cas de l'affrication, le «bruit» est limité à la partie supérieure du spectrogramme tandis que l'aspiration se manifeste sur l'ensemble des valeurs spectrales. De plus, la durée de l'affrication est habituellement au moins deux fois plus longue que celle de l'aspiration. Les caractéristiques de l'affrication du /t/ sont aisément reconnaissables dans l'affrication du /d/ tandis qu'un /t/ aspiré partage les mêmes caractéristiques que les /p/ et /k/ aspirés. 


\section{Résultats}

Cette section nous permettra de bien comparer l'affrication et l'aspiration chez nos quatre locuteurs. Nous avons fait une série de tableaux qui montrent l'importance de chaque phénomène examiné et les conditions propices à sa présence en comparant sa fréquence dans une variété de circonstances. Chaque phénomène est d'abord traité individuellement et puis nous considérons l'influence que la présence d'un phénomène peut avoir sur la présence d'un autre.

Le tableau suivant donne dans un premier temps la distribution générale des occlusives à l'étude pour les quatre locuteurs.

\begin{tabular}{|c|c|c|c|c|c|c|c|c|c|c|c|c|c|}
\hline Loc. & Cotal & $/ \mathbf{b} /$ & $\begin{array}{l}\% \\
\text { total }\end{array}$ & $/ \mathbf{d} /$ & $\begin{array}{l}\% \\
\text { total }\end{array}$ & $/ g /$ & $\begin{array}{l}\% \\
\text { total }\end{array}$ & $/ \mathbf{p} /$ & $\begin{array}{l}\% \\
\text { total }\end{array}$ & $\mid \mathbf{t} /$ & $\begin{array}{l}\% \\
\text { total }\end{array}$ & $/ \mathbf{k} /$ & $\begin{array}{l}\% \\
\text { total }\end{array}$ \\
\hline F1 & 717 & 53 & 7.39 & 209 & 2015 & 16 & 2.23 & 125 & 17.43 & 183 & 25.52 & 131 & 18.27 \\
\hline F2 & 28 & 26 & 9.22 & 77 & 30 & 9 & 3.19 & 56 & .86 & 63 & & 51 & 18.09 \\
\hline M1 & 93 & 45 & 4.83 & 228 & & 21 & 2.26 & 157 & 16.86 & 263 & & 217 & 23.31 \\
\hline M3 & 734 & 50 & 6.81 & 135 & 18.39 & 44 & 5.99 & 123 & 16.76 & 231 & 31.47 & 151 & 20.57 \\
\hline & 2664 & 174 & 7.06 & 649 & 24.83 & 90 & 3.42 & 461 & 17.73 & 740 & 26.90 & 550 & 20.06 \\
\hline
\end{tabular}

Tableau 1: Occurrences des occlusives. Nombre individuel et total de chaque occlusive, le pourcentage du phonème par locuteur et le pourcentage moyen pour chaque phonème.

Bien que la fréquence de chaque occlusive /b, d, g, p, t, k/ soit similaire entre les locuteurs (cf. tableau 1), il faut mentionner que le nombre total d'occurrences chez F2 est très réduit ( $282 \mathrm{vs} .717,931$ et 734 pour les autres), ce qui peut provoquer de fortes divergences en ce qui concerne la fréquence de chacun des phénomènes phonétiques à l'étude. Cette locutrice, âgée de 87 ans lors de l'entrevue, n'était pas aussi bavarde que les autres et l'intervieweur avait souvent besoin d'inciter des réponses plus élaborées. Cependant, elle a passé une bonne partie de sa vie soit vers la frontière entre les régions créole et cadienne, soit à l'extérieur de la région créole, et sa proximité aux Cadiens qui n'affriquent pas aurait pu influencer sa prononciation. Puisqu'il ne s'agit que d'un micro-corpus, où nous n'examinons qu'une partie (à peu près 10 minutes) d'une entrevue plus longue, des facteurs tels que la loquacité du locuteur et le sujet de la conversation peuvent avoir un impact sur la fréquence de chaque occlusive, la présence des phénomènes en question et le nombre d'anglicismes. Cela dit, le corpus est quand même assez vaste pour nous fournir un bon aperçu de la présence de ces phénomènes dans le parler de la Ville Platte.

\subsection{L'affrication}

Le pourcentage des /t/ et /d/ affriqués par chaque locuteur est similaire, mais le manque de données dans le cas de F2 pourrait avoir mené à une différence légère dans son cas. Le tableau 2 ne montre que les affriquées considérées comme françaises. Contrairement aux affriquées françaises, la majorité des affriquées anglaises sont des phonèmes (sauf dans le cas des groupes $/ \mathrm{tr} / \mathrm{et} / \mathrm{dr} /$ en position initiale de syllabe voir 2.1) et elles sont toutes palatalisées. Nous avons aussi mentionné en section 2.1 la présence de l'affrication alvéo-palatales devant les voyelles antérieures. Selon Picone et Valdman, ce type d'affriquée est caractéristique du système consonantique du FC (2005:151). Ces affriquées sont listées sous « affriquées cadiennes » dans le tableau 2.

Le tableau suivant montre que l'affrication à la Ville Platte est presque systématique. Trois des quatre locuteurs affriquent les $/ \mathrm{d} /$ et les $/ \mathrm{t} /$ dans des contextes affriquants plus de $89 \%$ du temps. Le cas de F2, qui n'affrique les /d/ que $72 \%$ du temps (mais les /t/ à 100\%) est une anomalie. 


\begin{tabular}{|c|c|c|c|c|c|c|c|}
\hline & Loc. & $\begin{array}{l}\text { Affriquées } \\
\text { possibles }\end{array}$ & $\begin{array}{l}\text { Nombre } \\
\text { affriqué }\end{array}$ & $\begin{array}{l}\% \\
\text { affriqué }\end{array}$ & $\begin{array}{l}\text { Affriquées } \\
\text { cadiennes }\end{array}$ & $\begin{array}{l}\% \text { des affriquées } \\
\text { qui sont cadiennes }\end{array}$ & $\begin{array}{l}\text { Affriquées } \\
\text { inattendues }\end{array}$ \\
\hline \multirow{4}{*}{$/ \mathbf{d} /$} & F1 & 39 & 38 & 97.44 & 0 & 0.00 & 0 \\
\hline & F2 & 11 & 8 & 72.73 & 2 & 25.00 & 1 \\
\hline & M1 & 69 & 62 & 89.86 & 5 & 8.06 & 1 \\
\hline & M3 & 34 & 34 & 100.00 & 5 & 14.71 & 1 \\
\hline \multirow{4}{*}{$/ \mathrm{t} /$} & F1 & 31 & 30 & 96.77 & 0 & 0.00 & 3 \\
\hline & F2 & 3 & 3 & 100.00 & 1 & 33.33 & 4 \\
\hline & M1 & 34 & 34 & 100.00 & 8 & 23.53 & 4 \\
\hline & M3 & 32 & 30 & 93.75 & 8 & 26.67 & 8 \\
\hline \multirow{4}{*}{ Total } & F1 & 70 & 68 & 97.14 & 0 & 0.00 & 3 \\
\hline & F2 & 14 & 11 & 78.57 & 3 & 27.27 & 5 \\
\hline & M1 & 103 & 96 & 93.20 & 13 & 13.54 & 5 \\
\hline & M3 & 66 & 64 & 96.97 & 13 & 20.31 & 9 \\
\hline
\end{tabular}

Tableau 2 : Les affriquées. Nombre d'affriquées possibles qui sont réalisées par locuteur. Inclus sont les $/ \mathrm{t} /$, les /d/ et le total des deux (l'affrication dans des mots considérés comme anglais est éliminée). Les deux colonnes centrales montrent le nombre et pourcentage de toutes les affriquées qui sont palatalisées (les affriquées «cadiennes ») par locuteur. La dernière colonne montre le nombre d'affriquées inattendues.

Le tableau nous montre que les pourcentages des affriquées sont très similaires parmi les locuteurs, sauf l'anomalie de F2, dont on a parlé plus haut. En nombre absolu, /d/ est plus affriqué que /t/ mais, en pourcentage /t/ est plus affriqué que /d/. En ce qui concerne les affriquées « cadiennes », ou les affriquées palatalisées, leur présence varie entre $0 \%$ et un tiers des occurrences en fonction de l'occlusive et du locuteur. Cette variation serait probablement plus stable dans un corpus plus grand. Une bonne partie du nombre élevé d'affriquées palatalisées chez certains locuteurs peut être attribuée à la répétition de mots tels que cadien(s) $[\operatorname{kad} 3(j) \tilde{\varepsilon}]$ (chez M1 et M3 : 3 et 4 fois respectivement), et dépend, alors, du sujet de discussion pendant l'extrait de 10 minutes utilisé dans cette étude.

Dans ce mini-corpus, les locuteurs ont parfois affriqué là où l'on ne s'attendrait pas à l'affrication, c'està-dire devant des voyelles non fermées ou non antérieures (cf. deux [dzø]). Nous constatons que les /t/ sont affriqués dans des contextes non affriquants considérablement plus souvent que les /d/, mais les affriquées inattendues représentent un nombre minuscule de toutes les occlusives cernées et un faible pourcentage de la totalité des affriquées (attendues et non attendues). Parfois des affriquées anglaises apparaissent dans des emprunts et sont considérées comme des affriquées (palatalisées) inattendues. Par exemple, dans le mot jogue [d3oひg] («jug »), le [d3] est inclus parmi les affriquées palatalisées bien que le phonème non affriqué auquel on s'attendrait en FS soit [3] et pas [d].

Avant de continuer il faut examiner de plus près le cas de M1. Chez ce locuteur il existe un groupe de mots considéré comme français, mais dans lequel il n'affrique pas le /d/ dans des conditions affriquantes. Il s'agit des mots " disque » (13 fois), « distribuer» (2 fois) et le nom propre Dugas ( 1 fois). Ces trois mots sont liés à sa carrière de producteur et vendeur de musique cadienne (Ivy Dugas est un musicien cadien), une industrie dans laquelle il est obligé d'utiliser l'anglais la plupart du temps. Les /d/ et /t/ dans ces mots ne sont pas considérés comme non-affriquées et ne figurent pas dans le tableau. D'autres mots liés à la vente de musique qui paraissent dans l'enregistrement et dans lesquels les conditions d'affrication sont remplies sont «C. D.», «D. J.», et «studio », mais ces mots étaient prononcés à l'anglaise et étaient considérés comme anglais. 


\subsubsection{L'accentuation et la position dans le mot}

En ce qui concerne les causes de l'affrication, il y a beaucoup de variation entre les locuteurs. Cette variation semble indiquer que l'affrication est plutôt le résultat du contexte phonétique que de la position dans un mot ou de l'accentuation. Toutefois, Poirier indique que «l'oreille perçoit mieux le phénomène en syllabe accentuée qu'en syllabe inaccentuée » (2009:378). Le pourcentage d'affriquées accentuées est beaucoup plus élevé dans des syllabes ailleurs dans le mot qu'au début. Cette dernière constatation découle probablement du fait que l'accentuation en français tombe sur la dernière syllabe du mot ou du groupe de mots.

\subsubsection{Le phénomène $x$}

Il existe, dans notre corpus, un autre phénomène qui ressemble à l'affrication, mais la partie fricative, qui dure plus longtemps qu'une explosion normale, est considérablement plus brève que celle d'une affriquée. Nous avons décidé de l'appeler le phénomène $x$. Dans un seul spectrogramme, la figure suivante compare le phénomène $\mathrm{x}$ (qui affecte le $[\mathrm{t}] \mathrm{du}$ mot « emprunter ») avec l'affrication (qui affecte le [t] du mot «outils»). Nous pouvons voir que la fréquence de la partie fricative $d u[t] d u$ mot « emprunter » descend de gauche à droite comme le $[\mathrm{t}]$ affriqué du mot « outils », mais cette descente est plus brève et est à une fréquence plus élevée.

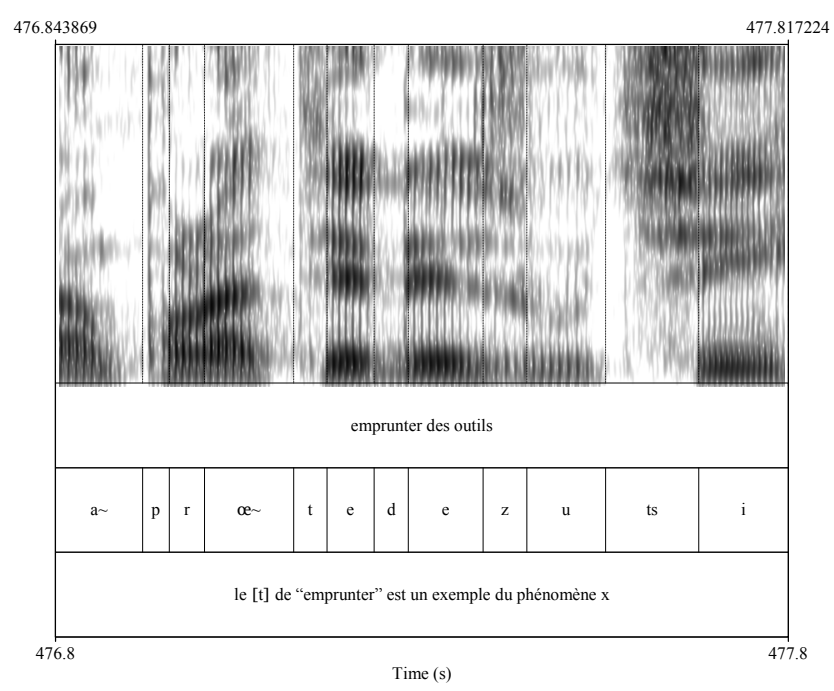

Figure 3 : Spectrogramme du phénomène $x$ et de l'affrication. Une comparaison du phénomène $x$ ([t] de « emprunter ») avec l'affrication ([t] de « outils»). (loc. M1, 477s)

Nous pouvons voir que la partie fricative du phénomène $\mathrm{x}$ est beaucoup plus courte que celle de l'affrication. Ce phénomène n'est observé qu'avec l'occlusive sourde [t] précédant les voyelles [e] et $[\mathrm{u}]$ (avec quelques rares exceptions comme $[\partial]$ et $[\mathrm{w}]$ ), ce qui n'est pas surprenant puisque Poirier mentionne que «le /t/ s'assibile plus nettement que le /d/» (2009:378). Le tableau suivant montre la fréquence du phénomène $\mathrm{x}$ dans notre mini-corpus. 


\begin{tabular}{|c|c|c|c|c|c|c|c|c|c|}
\hline & Loc. & Total & Phén. x & $\% \operatorname{des} / t /$ & [_e] & $\%$ [_e] & [_u] & $\%$ \% [_u] & autre voy \\
\hline \multirow{5}{*}{$/ \mathbf{t} /$} & F1 & 183 & 8 & 4.37 & 2 & 25.00 & 5 & 62.50 & 1 \\
\hline & $\mathrm{F} 2$ & 63 & 5 & 7.94 & 3 & 60.00 & 2 & 40.00 & 0 \\
\hline & M1 & 263 & 9 & 3.42 & 4 & 44.44 & 3 & 33.33 & 2 \\
\hline & M3 & 231 & 10 & 4.33 & 9 & 90.00 & 1 & 10.00 & 0 \\
\hline & & 740 & 32 & 5.01 & 18 & 54.86 & 11 & 36.46 & 3 \\
\hline
\end{tabular}

Tableau 3 : Phénomène $x$. Le pourcentage des /t/ qui démontrent le phénomène $x$. Le nombre et le pourcentage qui précèdent $[\mathrm{e}]$ et $[\mathrm{u}]$ et le nombre devant une autre voyelle.

Ce phénomène, rare chez tous les locuteurs, ne surviennent qu'avec environ $4 \%$ des /t/ (F2 fait exception encore une fois), et est réalisé un peu plus souvent devant [e] que devant [u]. Thomas a parlé d'un phénomène semblable dans son étude de la variation en franco-ontarien. En discutant des raisons pour lesquelles on affrique devant certaines voyelles il souligne que :

Ces différences proviennent de la nature palatalisante des voyelles les plus fermées et de la faible distance qui sépare celles-ci du point d'articulation des apico-dentales : la langue se décolle plus lentement de sa position occlusive que si elle devait se retirer jusqu'à la vélaire/u/, par exemple, et on peut alors entendre plus facilement les articulations intermédiaires. Il se peut également que la forme de la cavité de résonance en arrière du point d'occlusion joue un rôle appréciable: les cas d'assibilation devant le /u/ de «tout(es)» relevés par Marchal (1980) à Montréal le suggèrent fortement (1986:73).

L'affrication de /t/ a lieu devant les voyelles antérieures et fermées. En ce qui concerne le phénomène $\mathrm{x}$, dans la vaste majorité des cas, cette affrication légère a lieu devant des voyelles qui partagent au moins un de ces traits. La voyelle [u] est fermée (et postérieure), la voyelle [e] est antérieure (et mi-fermée), la semi-consonne [w] partage des traits avec [u], et nous avons constaté souvent dans les spectrogrammes de notre corpus que les formants du schwa ressemblent à ceux de la voyelle antérieure, fermée et relâchée [I] (affricable en français québécois) selon les tableaux offerts par Ladefoged (1996:100).

\subsection{L'aspiration}

Tel qu'expliqué dans la section 2.2, l'aspiration est un phénomène anglais qui est très commun dans le français de nos locuteurs. Cette présence est probablement le résultat du bilinguisme sociétal, puisqu'à part avec certains amis et membres de la famille, tous les francophones en Louisiane communiquent en anglais (Blyth 1997:30). L'aspiration est un phénomène qui n'affecte que les occlusives sourdes et nous allons comparer la fréquence d'aspiration dans les mots français et anglais.

Afin d'être aspirée, une occlusive sourde ne peut pas être précédée par /s/ (Crystal 2008 :38) et, bien que l'aspiration soit la plus forte au début des syllabes accentuées, nous considérons comme aspirable chaque $/ \mathrm{p} /$, / $/ \mathrm{t} /$ et $/ \mathrm{k} /$ qui n'est pas précédé par $/ \mathrm{s} /$ et qui est en attaque de syllabe. En ce qui concerne le /t/ à l'intérieur d'un mot anglais, il devient souvent une consonne alvéolaire battue $/ \mathrm{r} /$ devant une syllabe inaccentuée (Ladefoged 2005:71) et dans de tels cas nous ne le considérons pas comme aspirable. Nous n'avons pas non plus considéré les / $\mathrm{t}$ / affriqués et ceux qui manifestent les effets du phénomène $\mathrm{x}$ (cf. 4.1.2) comme aspirable. Nous avons aussi éliminé les rares exemples observés d'aspiration devant /1/ et $/ \mathrm{r} /$. Le tableau suivant montre le nombre et pourcentage d'aspirées dans les deux langues. 


\begin{tabular}{|c|c|c|c|c|c|c|c|c|}
\hline & Loc. & $\begin{array}{l}\text { Total } \\
\text { aspiré }\end{array}$ & $\begin{array}{l}\text { Aspirées } \\
\text { possibles } \\
\text { (français) }\end{array}$ & $\begin{array}{l}\text { Nombre } \\
\text { Aspiré } \\
\text { (français) }\end{array}$ & $\begin{array}{l}\text { \% } \\
\text { (français) }\end{array}$ & $\begin{array}{l}\text { Aspirées } \\
\text { possibles } \\
\text { (anglais) }\end{array}$ & $\begin{array}{l}\text { Nombre } \\
\text { Aspiré } \\
\text { (anglais) }\end{array}$ & $\begin{array}{l}\% \\
\text { (anglais) }\end{array}$ \\
\hline \multirow{4}{*}{$/ \mathbf{k} /$} & F1 & 53 & 92 & 45 & 48.91 & 9 & 8 & 88.89 \\
\hline & $\mathrm{F} 2$ & 17 & 39 & 14 & 35.90 & 4 & 3 & 75.00 \\
\hline & M1 & 55 & 140 & 46 & 32.86 & 10 & 9 & 90.00 \\
\hline & M3 & 41 & 122 & 41 & 33.61 & 1 & 0 & 0 \\
\hline \multirow{4}{*}{$/ \mathbf{p} /$} & F1 & 23 & 105 & 19 & 18.10 & 6 & 4 & 66.67 \\
\hline & $\mathrm{F} 2$ & 4 & 44 & 4 & 9.09 & 0 & 0 & NA \\
\hline & M1 & 17 & 108 & 16 & 14.81 & 3 & 1 & 33.33 \\
\hline & M3 & 18 & 102 & 18 & 17.65 & 0 & 0 & NA \\
\hline \multirow{4}{*}{$/ \mathbf{t} /$} & F1 & 22 & 74 & 14 & 18.92 & 10 & 8 & 80.00 \\
\hline & $\mathrm{F} 2$ & 6 & 18 & 6 & 33.33 & 0 & 0 & NA \\
\hline & M1 & 20 & 117 & 18 & 15.38 & 4 & 2 & 50.00 \\
\hline & M3 & 48 & 116 & 48 & 41.38 & 1 & 0 & 0 \\
\hline \multirow{4}{*}{ Total } & F1 & 98 & 271 & 78 & 28.78 & 25 & 20 & 80.00 \\
\hline & F2 & 27 & 101 & 24 & 23.76 & 4 & 3 & 75.00 \\
\hline & M1 & 92 & 365 & 80 & 21.92 & 17 & 12 & 70.59 \\
\hline & M3 & 107 & 340 & 107 & 31.47 & 2 & 0 & 0 \\
\hline
\end{tabular}

Tableau 4 : Les aspirées. Le pourcentage d'occlusives aspirées selon qu'elles sont françaises ou anglaises.

Il est clair que la fréquence d'aspiration dans ce corpus est beaucoup plus importante que la fréquence $\mathrm{d}$ 'affrication et que ce phénomène, typiquement anglais, a pénétré le français de la Ville Platte. Le / $\mathrm{k} /$ semble être plus susceptible à l'aspiration que les autres occlusives puisqu'il est le plus aspiré chez tous les locuteurs sauf M1. En ce qui concerne la présence de l'aspiration dans l'anglais de nos locuteurs, il existe une forte tendance à l'aspiration et cette tendance aurait vraisemblablement un impact sur leur français. Dans les sections qui suivent nous allons considérer l'impact de l'accentuation et la position dans le mot sur l'occlusive en question et si l'aspiration est en conflit avec l'affrication.

\subsubsection{L'accentuation et l'aspiration en français}

Puisque l'aspiration en anglais est bien documentée, dans cette section nous allons mettre l'accent sur l'aspiration dans les mots français afin de déterminer s'il y a des liens entre cette aspiration et l'accentuation et/ou la position de l'occlusive dans le mot. Pour les aspirées au début du mot, le pourcentage accentué fluctue entre un nombre bien en dessous de la moitié et les deux tiers. Par contre, une grande majorité des aspirées qui ne sont pas au début des mots sont dans des syllabes accentuées chez tous les locuteurs (67 à 100\%). Tel que remarqué pour l'affrication (cf. section 4.1), ceci semble normal puisque l'accentuation française tombe sur la dernière syllabe du mot (ou groupe de mots) et la longueur de la phrase phonologique en français louisianais est nettement plus courte qu'en français standard, permettant au locuteur d'accentuer chaque unité lexicale (voir la section 2.3) (Lyche 1996:42).

\subsection{Les deux phénomènes en combinaison}

Dans cette section nous considérons si les deux phénomènes en question dans cette étude, peuvent s'influencer. Nous n'avons constatée la combinaison de l'aspiration et de l'affrication qu'une seule fois (d'autres cas possibles étant difficiles à confirmer). De plus, l'affrication dans ce cas a eu lieu dans un contexte qui n'est pas normalement propice à l'affrication (le mot «temps » [ts/hã]) et était même unique parmi nos affriquées inattendues (cf. la section 4.1). Ces deux facteurs indiquent que c'est un phénomène 
très rare et nous n'allons pas généraliser sans plus d'occurrences. Globalement, l'affrication semble empêcher l'aspiration. Nous avons vu plus haut que l'affrication était presque systématique dans le dialecte en question (cf. 4.1) et nous n'avons pas vu d'aspiration dans un contexte affriquant.

\section{Discussion et conclusions}

Nos résultats mettent en lumière que l'affrication est toujours importante chez nos locuteurs malgré les influences de l'anglais et des autres dialectes français de la Louisiane. La pénétration de l'aspiration (un trait typiquement anglais) montre que l'anglais exerce une influence sur cette variété de français, mais le fait que l'affrication est toujours quasi-systématique dans les mots français révèle que la tendance à l'affrication parmi nos locuteurs est encore assez enracinée pour résister à toutes les influences qui viennent de l'extérieur de la communauté. Nos résultats semblent confirmer que ces deux phénomènes, l'affrication et l'aspiration, s'excluent mutuellement. D'autres phénomènes tels que la palatalisation n'étaient pas assez fréquents dans notre mini-corpus pour faire une analyse plus approfondie.

Nous avons vu qu'il existe des différences entre les locuteurs en ce qui concerne la fréquence de certains phénomènes, mais, si nous considérons le fait que les enregistrements ne sont pas assez longs pour éliminer des anomalies statistiques potentielles, nous pouvons voir que globalement les locuteurs ont à peu près les mêmes taux d'affrication et d'aspiration. En ce qui concerne les différences entre les locuteurs sur le plan de la grammaire et du choix des emprunts à l'anglais, il y a vraisemblablement des indices qui indiqueraient une tendance à des grammaires individuelles, c'est-à-dire que les francophones louisianais sont si peu nombreux et utilisent le français si rarement que chacun a sa propre grammaire, mais cela dépasse la portée de cette recherche.

Puisque ces locuteurs sont tous âgés de plus de soixante ans, dans des recherches futures il serait intéressant de comparer nos statistiques avec celles d'un groupe de francophones plus jeunes afin de déterminer si l'affrication est toujours aussi puissante et si l'aspiration a commencé à la remplacer dans certaines circonstances. Dubois et Horvath (1998:246) ont mentionné dans leur étude de la dentalisation des fricatives interdentales en anglais cadien (AC) que la non-aspiration des occlusives $/ \mathrm{p}, \mathrm{t}, \mathrm{k} /$ est typique de l'AC, mais nous avons montré qu'à la Ville Platte, l'influence interlinguistique agit dans l'autre direction et que ces occlusives sont aspirées en français. Si cette influence continue de s'accroître et s'il y a un affaiblissement de la tendance à l'affrication chez des locuteurs plus jeunes, il est possible que l'aspiration remplace l'affrication devant [i] et [j] (et peut-être [y] et [u] qui ne sont pas des phonèmes anglais). Nous avons vu chez M1 que les locuteurs sont capables de ne pas affriquer dans des circonstances affriquantes, mais la majorité du temps la non-affrication ne s'applique qu'à un groupe spécifique de mots. En comparant les générations il faut aussi considérer le nivellement des dialectes ainsi que l'étiolement de plus en plus important du français en Louisiane et leur impact phonétique sur la langue.

\section{Références bibliographiques}

Blyth, C. (1997). The Sociolinguistic Situation of Cajun French : The Effects of Language Shift and Language Loss. Dans A. Valdman, French and Creole in Louisiana (pp. 25-46). New York: Plenum Press.

Boersma, P., \& Weenik, D. (2009). Praat: Doing phonetics by computer. http://www.fon.hum.uva.nl/praat/.

Corneau, C. (2000). An EPG study of palatalization in French: Cross-dialect and inter-subject variation. Language Variation and Change, 12, 25-49.

Crystal, D. (2008). A Dictionary of Lingistics and Phonetics (6th ed.). Malden, MA: Blackwell Publishing.

Daigle, J. O. (1993). A Dictionary of the Cajun Language. Ville Platte, LA: Swallow Publications.

Davenport, M., \& Hannahs, S. (1998). Introducing Phonetics and Phonology. New York: Arnold Publishers. 
Dubois, S. (2005). Un siècle de français cadien parlé en Louisiane : persistance linguistique, hétérogénéité géographique et évolution. Dans A. Valdman, J. Auger, \& D. Piston-Hatlen, Le français en Amérique du Nord: état présent (pp. 287-305). Québec: Presses de l'Université Laval.

Dubois, S., \& Horvath, B. M. (1998). Let's tink about dat: Interdental fricatives in Cajun English. Language Variation and Change, 10, 245-261.

Dubois, S., Noetzl, S., \& Salmon, C. (2006). L'usage des pratiques bilingues dans la communauté cadienne. Revue Canadienne de Linguistique Appliquée, 9 (2), 207-219.

Klingler, T. A., Picone, M. D., \& Valdman, A. (1997). The Lexicon of Louisiana French. Dans A. Valdman, French and Creole in Louisiana (pp. 145-181). New York: Plenum.

Ladefoged, P. (1996). Elements of acoustic phonetics. Chicago: University of Chicago Press.

Ladefoged, P. (2003). Phonetic Data Analysis. Malden, MA: Blackwell Publishing.

Ladefoged, P. (2005). Vowels and Consonants: An Introduction to the Sounds of Languages. Malden, MA: Blackwell Publishing.

Laver, J. (1994). Principles of Phonetics. Cambridge, UK: Cambridge University Press.

Leclerc, J. (1989). Qu'est-ce que la langue? (2e ed.). Saint-Jérôme, QC: Mondia Éditeurs.

Lyche, C. (1996). Genèse et traits caractéristiques du français cadien : un aperçu phonologique. Revue Romane , 31 (1), 29-49.

Papen, R. A., \& Rottet, K. J. (1997). A Structural Sketch of the Cajun Spoken in Lafourche and Terrebonne Parishes. Dans A. Valdman, French and Creole in Louisiana (pp. 71-108). New York: Plenum.

Picone, M. D., \& Valdman, A. (2005). La situation du français en Louisiane. Dans A. Valdman, J. Auger, \& D. Piston-Hatlen, Le français en Amérique du Nord : État présent (pp. 143-165). Québec: Presses de l'Université Laval.

Poirier, C. (2009). L'Assibilation des occulusives /t/ et /d/ au Québec: le point sur la question. Dans L. Baronian, \& F. Martineau, Le Français d'un continent à l'autre (pp. 375-419). Québec: Presses de l'Université Laval.

Thomas, A. (1986). La variation phonétique : le cas du franco-ontarien. LaSalle, QC: Didier. 